\title{
Litíase em Rim em Ferradura em Doente com Ureter Reimplantado: A Propósito de
} um Caso Clínico

\section{Urinary Stones in a Horseshoe Kidney with Ipsilateral Ureteral Reimplantation: Case Report}

\author{
Diogo Nunes-Carneiro ${ }^{1 *}$, Rui Oliveira ${ }^{2}$ Vítor Cavadas $^{1}$
}

\section{Resumo}

O rim em ferradura é uma anomalia renal congénita muito comum, estando muitas vezes associada a infeções do trato urinário e a litíase renal como consequência das alterações anatómicas que dificultam uma drenagem eficaz de urina. 0 tratamento dos cálculos nestes casos torna-se um desafio devido às anomalias anatómicas subjacentes exigindo uma adaptação das técnicas normalmente utilizadas para o tratamento da litíase renal.

O artigo reporta o caso de uma mulher de 44 anos, com uma história de reimplantação ureteral à esquerda do tipo Cohen por refluxo vesico-ureteral na infância e com litíase renal. A doente apresentava dois cálculos com 12 e 10 mm de maior diâmetro no pielão superior do hemi-rim esquerdo, cujo infundíbulo tinha um calibre estreito. Procedeu-se a uma ureterorrenoscopia flexível com litotrícia laser Holmium através da colocação de uma baínha ureteral acima do infundíbulo estreitado tendo a doente ficado sem cálculos no final do procedimento.

Torna-se importante, nos casos de litíase em rim em ferradura, pensar e adaptar as várias técnicas disponíveis para o tratamento da litíase renal e eleger aquela que, à partida, proporcionará uma maior taxa de sucesso com menos complicações.

Palavras-chave: Cálculos Renais; Obstrução Ureteral; Reimplante; Rim/anomalias congénitas

\section{Introdução}

O rim em ferradura é das anomalias renais congénitas mais frequentes, tendo uma incidência estimada de 1 caso em cada 400-1600 nascimentos. Esta malformação caracteriza-se por um defeito de rotação, que se associa a uma grande variação na vascularização do rim, a uma inserção alta do ureter e a estenose da junção ureteropiélica em um terço dos casos. Devido

${ }^{1}$ Serviço de Urologia, Centro Hospitalar e Universitário do Porto, Porto, Portugal

${ }^{2}$ Serviço de Urologia, Centro Hospitalar de Entre o Douro e Vouga, Santa Maria da Feira, Portugal

\section{Abstract}

Horseshoe kidney is a common congenital renal anomaly and is often associated with urinary tract infections and renal calculi as a consequence of anatomical changes that impair the efficient drainage of the urine. The treatment of urinary stones in these cases becomes a challenge due to the underlying anatomical abnormalities requiring an adaptation of the techniques normally used for the treatment of renal lithiasis.

We report the case of a 44-year-old woman with a past history of Cohen left ureteral reimplantation due to vesico-ureteral reflux in childhood and renal lithiasis. The patient had two 12 and $10 \mathrm{~mm}$ stones in the upper calix of the left hemi-kidney, with a narrow infundibulum.

A flexible ureteroscopy with laser Holmium lithotripsy was performed by placement of a ureteral sheath over the narrowed infundibulum and the patient was left stone-free at the end of the procedure.

It is important, in cases of horseshoe kidney stones, to think and adapt the various techniques available for the treatment of renal lithiasis, and to choose the one that will provide a higher success rate with fewer complications.

Keywords: Kidney/abnormalities; Kidney Calculi; Replantation; Ureteral Obstruction

a estas alterações anatómicas a drenagem urinária é afetada, aumentando o risco de infeções e de litíase renal. ${ }^{1,2}$

A litíase é um fenómeno comum em doentes com esta patologia, tendo uma frequência que varia entre os $20 \%$ e os $80 \%$. $^{1,3}$ Apesar da explicação mais frequente para este fenómeno ser o facto da drenagem da urina estar prejudicada pelas alterações anatómicas destes rins, o mecanismo exato pelo qual os cristais se organizam e os cálculos se formam ainda não é claro. ${ }^{1,3}$

O tratamento dos cálculos torna-se um desafio nestes casos devido às anomalias estruturais que dificultam o acesso ao aparelho excretor sendo a taxa de sucesso inferior quando comparada com rins anatomicamente normais para o mesmo procedimento. ${ }^{4}$ 


\section{Caso Clínico}

É apresentado o caso de uma mulher de 44 anos, hipertensa e com história de cirurgia de reimplantação do ureter esquerdo na infância por refluxo vesico ureteral.

Recorreu à consulta por queixas de dor lombar à esquerda, com irradiação anterior com 2 semanas de evolução sem história de febre, hematúria ou sintomas urinários baixos.

Analiticamente não apresentava elevação dos parâmetros inflamatórios e a função renal encontrava-se preservada.

A ecografia revelou a presença de um rim em ferradura com ectasia calicial à esquerda causada por focos litiásicos, o maior com $9 \mathrm{~mm}$.

$\mathrm{Na}$ tomografia computorizada foram descritos no polo superior do hemi-rim esquerdo dois focos litiásicos com 12 e 10 mm de diâmetro com densidade média de 1000 HU, condicionando ligeira proeminência calicial. O cólon encontrava-se em posição retro-renal (Fig. 1) e observou-se ainda uma calcificação grosseira na raiz do mesentério com $2,4 \mathrm{~cm}$, traduzindo uma provável adenopatia calcificada.

Dada a história de cirurgia de reimplantação do ureter esquerdo foi feita uma cistoscopia tendo sido possível identificar o neomeato ureteral esquerdo no trígono vesical, à direita e em posição medial ao meato ureteral direito levantando-se a hipótese da doente ter sido submetida a uma reimplantação uretero-vesical tipo Cohen.

Foi proposta uma ureterorrenoscopia flexível com fragmentação dos cálculos a laser Holmium.

Iniciou-se o procedimento com a introdução de um fio-guia hidrofílico de corpo rígido até ao aparelho excretor alto guiado por fluoroscopia. A pielografia retrógrada revelou um atraso no preenchimento dos grupos caliceais médio e superior do hemirim esquerdo (Fig. 2). Durante a endoscopia constatou-se que o infundíbulo que daria acesso aos cálices em questão tinha um calibre pequeno sendo difícil fazer passar o aparelho em segurança. Foi colocado um segundo fio-guia de segurança e colocada a bainha ureteral acima do aperto, permitindo o acesso aos cálices (Fig. 3). Os cálculos foram identificados e fragmentados com fibra laser Holmium $200 \mu \mathrm{m}$ com uma energia média de 0,6 J e uma frequência média de $14 \mathrm{~Hz}$ num total de 20497 disparos. Os fragmentos foram removidos com um cesto de $1.3 \mathrm{Fr}$ com $120 \mathrm{~cm}$ de comprimento.

No final foi possível inspecionar todo o aparelho excretor incluindo o ureter, não tendo sido identificados fragmentos de tamanho significativo. Foi deixado um cateter ureteral duplo $\mathrm{J}$ $6 \mathrm{Fr}, 26 \mathrm{~cm}$ que foi removido quatro semanas após a cirurgia.

O procedimento durou 1 hora e 15 minutos, sendo que o pós-operatório decorreu sem intercorrências. A doente teve alta 24 horas após o procedimento.

$\mathrm{Na}$ tomografia computorizada (TC) de reavaliação realizada três meses após o procedimento já não havia referencia a litíase renal.

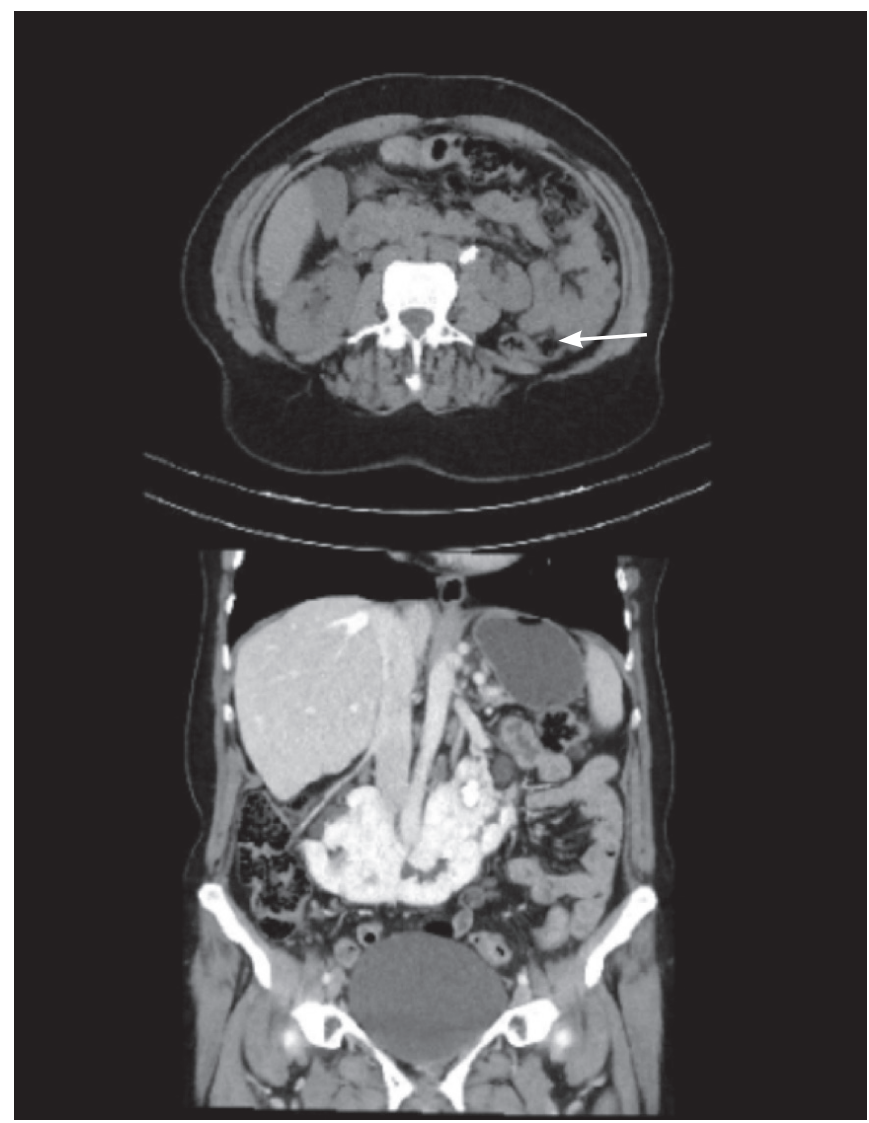

Figure 1: TC evidenciando a presença de cólon retro-renal (seta) e dois focos litiásicos com 12 e 10 mm de diâmetro com densidade média de $1000 \mathrm{HU}$ no hemi-rim esquerdo (ponta de seta).

\section{Discussão}

A litíase em rim em ferradura apresenta-se como um desafio cirúrgico. O estudo pré-operatório destes doentes deve ser aprofundado, nomeadamente através da realização de tomografia computorizada, de modo a caracterizar anatomicamente o rim e a eleger a melhor opção terapêutica tendo em conta as limitações das várias abordagens possíveis.

A litotrícia extracorporal por ondas de choque tem uma eficácia no tratamento da litíase em rins em ferradura que varia entre os $14,8 \%$ e $85,2 \% .{ }^{5} \mathrm{~A}$ maior limitação desta técnica prende-se com a anatomia do rim que dificulta a expulsão espontânea dos fragmento. ${ }^{6}$ Nesta situação em particular, o volume dos cálculos e a densidade fariam prever uma baixa taxa de sucesso associada a esta técnica, pelo que não foi

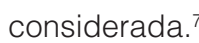

A nefrolitotomia percutânea é uma das técnicas mais frequentemente recomendadas no tratamento da litíase de rim em ferradura e está preconizada para doentes com cálculos de dimensões superiores a $20 \mathrm{~mm}$ ou para doentes que não responderam ao tratamento com litotrícia extracorporal por ondas de choque. ${ }^{8}$ Nos rins em ferradura, devido à má-ro- 


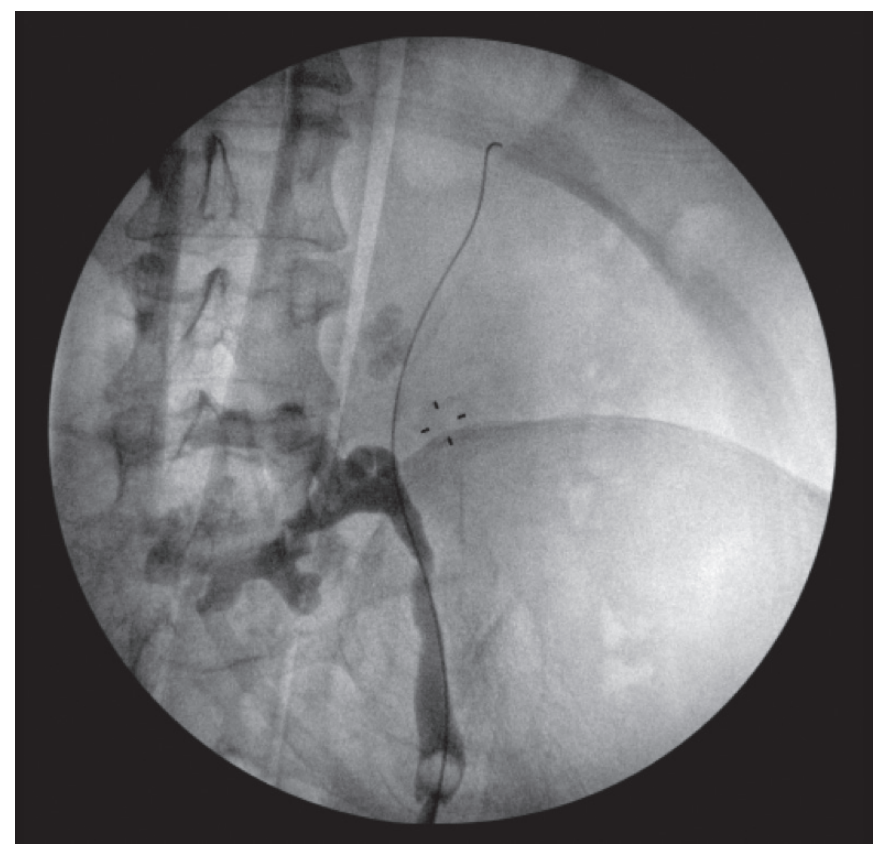

Figure 2: Pielografia retrógrada esquerda. Verifica-se onde se verifica o correto posicionamento do fio-guia no aparelho excretor alto e um atraso no preenchimento dos grupos caliceais médio e superior do hemi-rim esquerdo.

tação dos hemi-rins, os cálices encontram-se posicionados postero-inferiormente o que torna o acesso percutâneo mais vantajoso, sobretudo quando se opta por uma punção através do cálice superior, sendo um trajeto intercostal raramente necessário. ${ }^{6}$ Neste caso específico a localização retrorenal do cólon impedia, à partida, o acesso anterógrado. Caso a abordagem anterógrada fosse imperativa poder-se-ia rebater o cólon através de retroperitoneoscopia, ${ }^{9}$ sendo possivel realizar um acesso ao cálice sob visão. De modo a minimizar a lesão de órgãos vizinhos é fundamental a realização de ecografia intra-operatória em todos os procedimentos antes da criação do acesso.

No caso desta doente, a abordagem retrógrada seria uma opção válida e a história de reimplantação do ureter, mesmo constituindo um desafio técnico, não impediria o uso desta técnica. ${ }^{10}$ Apesar de os estudos serem escassos, a taxa de sucesso da ureterorrenoscopia varia entre $70 \%$ - 88,2\% ${ }^{11,12}$ tendo vindo a ser cada vez mais utilizada no tratamento desta patologia. ${ }^{13}$ Nesta cirurgia pode haver dificuldade na extração de todos os fragmentos sendo, por vezes, necessário um segundo procedimento para remover toda a massa litiásica. Mesmo assim, com o desenvolvimento de aparelhos de menor calibre e dispositivos que permitem a remoção de fragmentos, esta técnica tem vindo a ganhar uma importância crescente. ${ }^{14}$

Em doentes com deformidades anatómicas complexas e com cálculos de grande volume a cirurgia laparoscópica ou robótica podem ser opções a ter em conta, no entanto,

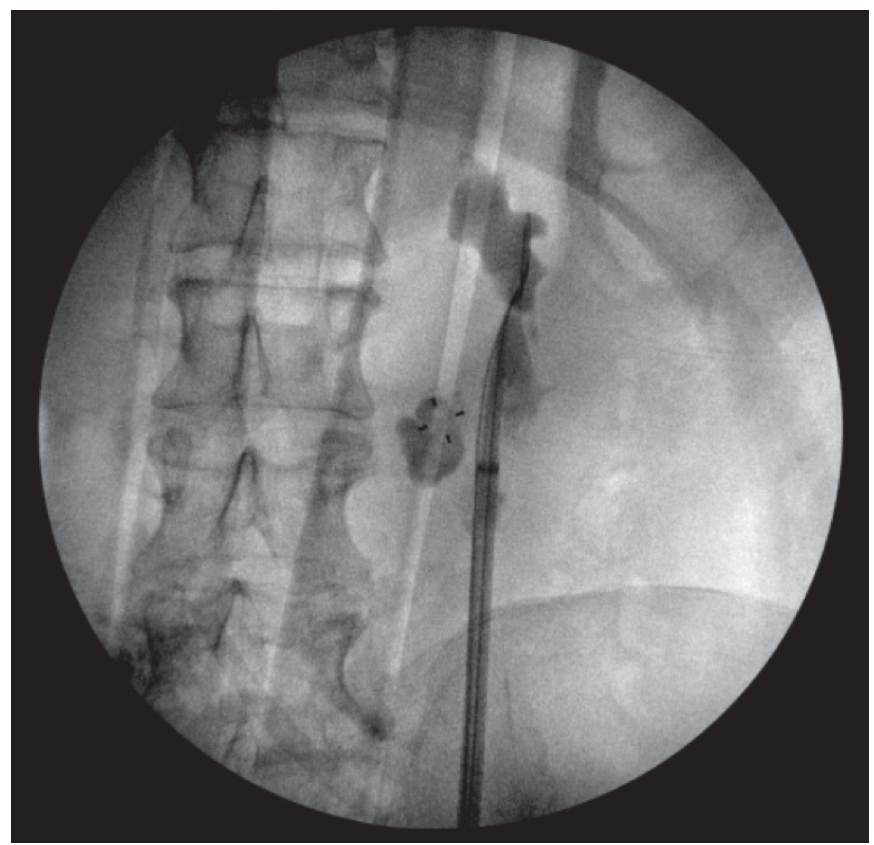

Figure 3: Posicionamento da bainha ureteral acima do infundíbulo estenótico permitindo assim a fragmentação do cálculoo tratamento do cálculo com sucesso e impedindo a migração dos fragmentos para outros cálices.

a taxa de complicações é significativamente superior quando comparadas com as técnicas descritas acima. ${ }^{9} \mathrm{~A}$ cirurgia robótica no tratamento da litíase renal está ainda em fase de implementação e ainda não existem estudos randomizados que comparem a eficácia desta técnica com as restantes disponíveis. ${ }^{15}$

Independentemente da técnica, nos doentes com rim em ferradura, é fundamental um estudo imagiológico pré-operatório de modo a prever possíveis dificuldades. É também importante o domínio das várias técnicas cirúrgicas e do armamentário cirúrgico à disposição de modo a poder-se optar por aquela que melhor se adequa ao doente em questão. Tais procedimentos devem, portanto, ser realizados por equipas experientes e em centros de grande volume.

Responsabilidades Éticas

Conflitos de interesse na realização do presente trabalho.

Fontes de Financiamento: Não existiram fontes externas de financiamento para a realização deste artigo.

Proteção de Pessoas e Animais: Os autores declaram que os procedimentos seguidos estavam de acordo com os regulamentos estabelecidos pelos responsáveis da Comissão de Investigação Clínica e Ética e de acordo com a Declaração de Helsínquia da Associação Médica Mundial.

Confidencialidade dos Dados: Os autores declaram ter seguido os protocolos do seu centro de trabalho acerca da publicação dos dados de doentes.

Ethical Disclosures

Conflicts of Interest: The authors report no conflict of interest. 
Funding Sources: No subsidies or grants contributed to this work.

Protection of Human and Animal Subjects: The authors declare that the procedures followed were in accordance with the regulations of the relevant clinical research ethics committee and with those of the Code of Ethics of the World Medical Association (Declaration of Helsinki).

Confidentiality of Data: The authors declare that they have followed the protocols of their work center on the publication of patient data.

*Corresponding Author $/{ }^{\star}$ Autor Correspondente:

Diogo Nunes-Carneiro, MD

Serviço de Urologia do Centro Hospitalar e Universitário do Porto Largo Prof. Abel Salazar, 1

4099-001 Porto

E-mail: diogocarneiro.urologia@chporto.min-saude.pt

Recebido/Received: 2017-12-04

Aceite/Accepted: 2018-10-07

\section{REFERENCES}

1. Ishii H, Rai B, Traxer O, Kata SG, Somani BK. Outcome of ureteroscopy for stone disease in patients with horseshoe kidney: Review of world literature. Urol Ann. 2015;7:470-4. doi: 10.4103/0974-7796.157969.

2. Weizer AZ, Silverstein AD, Auge BK, Delvecchio FC, Raj G, Albala DM, et al. Determining the incidence of horseshoe kidney from radiographic data at a single institution. J Urol. 2003:170:1722-6.

3. Muttarak M, Sriburi T. Congenital renal anomalies detected in adulthood. Biomed Imaging Interv J. 2012;8:7. doi: 10.2349/biij.8.1.e7.

4. Liatsikos EN, Kallidonis P, Stolzenburg JU, Ost M, Keeley F, Traxer O, et al. Percutaneous management of staghorn calculi in horseshoe kidneys: A multi-institutional experience. J Endourol. 2010;24:531-6. doi: 10.1089/ end.2009.0264

5. Gómez Pascual JA, Soler Martínez J, García Galisteo E, Díaz Ramírez F, Alvarado Rodríguez A, Blanco Reina F, et al. Extracorporeal shock-wave lithotripsy as treatment of lithiasis in horseshoe kidney. Arch Esp Urol. 2003;56:39-44

6. Prakash G, Sinha RJ, Jhanwar A, Bansal A, Sigh V. Outome of percutaneous nephrolithotomy in anomalous kidney: is it different? Urol Ann. 2017;9:23-26. doi: 10.4103/0974-7796.198836.

7. Gukuk A, Uyeturk U. Usefulness of hounsfield unit and density in the assessment and treatment of urinary stones. World J Nephrol. 2014;6:282-6. doi: 10.5527/wjn.v3.i4.282.

8. 8. Etemadian M, Maghsoudi R, Abdollahpour V, Amjadi M. Percutaneous nephrolithotomy in horseshoe kidney. Our 5-year experience. Urol J. 2013;10:856-60

9. Jiang K, Tang K, XuH, Chen H, Chen Z. Retroperitoneoscopy technique-assisted percutaneous nephrolithotomy for complexity horseshoe kidney with renal stones. Urol Int. 2016;97:285-291. doi: 10.1159/000446120.

10. Emiliani E, Michele T, Audouin M, Traxer O. Modern flexible ureteroscopy in Cohen cross-trigonal ureteral reimplantations. J Pediatr Urol. 2017;13:329-31. doi: 10.1016/j.jpurol.2017.03.009

11. Molimard B, Al-Qahtani S, Lakmichi A, Sejiny M, Gil-Diez de Medina S, Carpentier X, et al. Flexible ureterorenoscopy with holmium laser in horseshoe kidneys. Urology. 2010;76:1334-7. doi: 10.1016/j.urology.2010.02.072.

12. Atis G, Resorlu B, Gurbuz C, Arikan O, Ozyuvali E, Unsal A, et al. Retrograde intrarenal surgery in patients with horseshoe kidneys. Urolithiasis. 2013:41:79-83. doi: 10.1007/s00240-012-0534-7.

13. Gokce MI, Tokatli Z, Suer E, Hajiyev P, Akinci A, Esen B. Comparison of shock wave lithotripsy (SWL) and retrograde intrarenal surgery (rirs) for treatment of stone disease in horseshoe kidney patients. Int Braz J Urol. 2016;41:96-100. doi: 10.1590/S1677-5538.IBJU.2015.0023.

14. Ding J, Huang Y, Gu S, Chen Y, Peng J, Bai Q, et al. Flexible ureteroscopic management of horseshoe kidney renal calculi. Int Braz J Urol. 2015;41: 683-9. doi: 10.1590/S1677-5538.IBJU.2014.0086.

15. Ganpule AP, Prashant J, Desai MR. Laparoscopic and robot-assisted surgery in the management of urinary lithiasis. Arab J Urol. 2012;10:32-9. doi: 10.1016/j.aju.2011.12.003. 